\title{
Assistência de enfermagem no atendimento pré-hospitalar a vítimas de queimaduras: scoping review
}

\author{
Nursing in pre-hospital care for burn victims: a scoping review
}

Egresados de enfermería y sus concepciones sobre el mundo del trabajo
Rosivânia Ingrid Medeiros da Silval ${ }^{\oplus} ;$ Eloysa dos Santos Oliveiral'@; Rodrigo Rhuan Andrade Rocha' ${ }^{\prime}$;

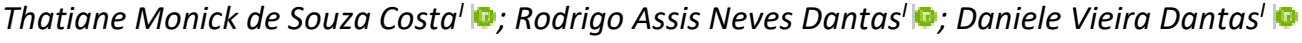

'Universidade Federal do Rio Grande do Norte, Natal, RN, Brasil

\begin{abstract}
RESUMO
Objetivo: identificar as evidências científicas sobre a assistência de enfermagem no atendimento pré-hospitalar a vítima de queimadura. Método: revisão de escopo realizada conforme orientações do Instituto Joanna Briggs e protocolo PRISMA-ScR. A busca foi executada em seis fontes de dados, no recorte temporal de 2016 a 2020. Doze artigos atenderam aos critérios de inclusão, sendo selecionados para a amostra dos resultados. Resultados: no âmbito da assistência de enfermagem, destacaramse reposição volêmica com infusão de fluidos; resfriamento local com água limpa, corrente e em temperatura ambiente; monitoramento/controle dos sinais vitais, uso de oxigenoterapia; analgesia e cuidados com a ferida, utilizando coberturas oclusivas, identificação do agente causador, avaliação da Superfície Corporal Queimada, extensão e profundidade da lesão. Conclusão: a assistência de enfermagem frente ao atendimento pré-hospitalar às vítimas de queimaduras se dá principalmente pelo controle da evolução dos danos causados pelo incidente desde o momento da chegada até o atendimento terciário. Descritores: Enfermagem; Assistência à Saúde; Serviços Médicos de Emergência; Queimaduras.
\end{abstract}

\section{ABSTRACT}

Objective: to identify the scientific evidence on nursing in pre-hospital care for burn victims. Method: this scoping Review was conducted following the Joanna Briggs Institute guidelines and the PRISMA-SCR protocol. Six data sources were searched in the time frame from 2016 to 2020. Twelve articles met the inclusion criteria and were selected for sampling of the results. Results: prominent within the scope of nursing care were fluid volume replacement by infusion; local cooling with clean, running water at room temperature; monitoring / control of vital signs, use of oxygen therapy; analgesia and wound care, using occlusive dressings, identification of the causative agent, assessment of the Burned Body Surface Area, extent and depth of the injury. Conclusion: nursing in pre-hospital care for burn victims mainly involves controlling the progression of the injury caused by the incident between the moment of arrival and tertiary care.

Descriptors: Nursing; Delivery of Health Care; Delivery of Health Care; Burns.

\section{RESUMEN}

Objetivo: identificar y analizar los aspectos facilitadores y limitantes de los estudiantes de enfermería en lo relativo a su inserción profesional. Método: estudio cualitativo y descriptivo, desarrollado en dos universidades federales de Río de Janeiro. Los participantes fueron 43 egresados de estas universidades, en los que se aplicó un guion de entrevista semiestructurada. El tratamiento de datos se realizó mediante análisis léxico con la ayuda del software Iramuteq. Resultados: se verificó que, en la Universidad A, los factores facilitadores para la realización del trabajo fueron: autonomía, relaciones interpersonales y equipo de trabajo. Los limitadores se caracterizan por: sobrecarga de trabajo, escasez de recursos humanos y de materiales, calificación profesional y poco reconocimiento. En la Universidad B, los factores facilitadores fueron: condiciones de trabajo, trabajo en equipo y autonomía. Los limitadores fueron: sobrecarga de trabajo, escasez de recursos humanos y de materiales, y relaciones interpersonales. Conclusión: cabe señalar que los factores dificultadores sobrepasaron los facilitadores e intervenciones y reformulaciones son necesarias, no solo en el ámbito laboral, sino también en el campo de la formación.

Descriptores: Enfermería; Prestación de Atención de Salud; Servicios Médicos de Urgencia Quemaduras.

\section{INTRODUÇÃO}

Queimaduras são danos traumáticos decorrentes de exposição a agente externo, seja de origem térmica, química, biológica, elétrica ou radioativa. São classificados quanto ao grau de comprometimento tissular e extensão de superfície atingida ${ }^{1}$.

Quanto ao comprometimento tissular, as queimaduras são classificadas em quatro graus. Queimaduras de primeiro grau atingem apenas a camada superficial da pele, caracterizadas por hiperemia e dor local. As de segundo grau são mais profundas, destruindo também parte da derme e caracterizam-se por flictemas, mantendo-se a dor na zona afetada. Distingue-se a queimadura de terceiro grau das outras a danificação da hipoderme, além da epiderme e derme, e é evidenciada comumente com a presença de lesões enegrecidas e dor em intensidades variadas. Por fim, as 
queimaduras de quarto grau são mais debilitantes e deformantes fisicamente, uma vez que atingem o máximo de profundidade tecidual possível, podendo chegar inclusive nos órgãos internos e outros sistemas ${ }^{2}$.

É importante reconhecer a extensão da queimadura para orientar as decisões durante o tratamento. Para isso, nos adultos, pode ser utilizada a da Regra dos nove, na qual cabeça, membros superiores, tórax e abdômen valem (9\%) cada, membros inferiores (18\%) e genital (1\%). Em pediatria, a classificação sofre adaptação sendo cabeça (18\%), membros superiores (9\%), tórax (9\%), abdômen (9\%), membros inferiores (13,5\%) e genital (1\%). Outra questão é que a queimadura pode mudar de características nas primeiras horas, sendo importante a observação constante e não tomar medidas precipitadas².

A Organização Mundial de Saúde (OMS) afirma que as queimaduras são um problema global de saúde pública. Estima-se que, mundialmente, ocorram cerca de 265.000 mortes/ano decorrentes de incêndios, além dos demais tipos de queimaduras, sejam elas elétricas ou de qualquer outra natureza ${ }^{3}$. No Brasil, ocorrem em média 1.000.000 de incidentes por queimaduras/ano. Destes, apenas 100.000 procuram atendimento hospitalar e dessa parcela, estima-se que 2.500 vítimas morrem direta ou indiretamente por consequência de suas lesões ${ }^{4}$.

As queimaduras ocorrem principalmente em casa ou no local de trabalho. Mulheres e crianças estão mais propensas a sofrerem queimaduras dentro de casa, geralmente na cozinha, decorrente de líquidos quentes ou chamas diretas, bem como explosões de fogões. Já com os homens é mais provável que sofram queimaduras no local de trabalho, em decorrência de incêndios, queimaduras químicas e elétricas. Dos sobreviventes, milhões ficam com deficiências e desfigurações ao longo da vida, muitas vezes atrelados ao estigma e preconceito social ${ }^{5}$.

Para atuar no cuidado às vítimas de queimaduras, o Atendimento Pré-Hospitalar (APH) móvel ou fixo tem por finalidade a agilidade assistencial e o raciocínio clínico apurado, de modo a minimizar o máximo de consequências significativas decorrentes da ocorrência ${ }^{6}$. No Brasil, ainda não é investido o necessário nos profissionais do APH. Muitos não possuem a capacitação permanente necessária ou não dispõem de estrutura mínima para operar, sem falar no estresse e tensão a que esses profissionais estão submetidos ${ }^{7}$.

Na maioria das vezes, a enfermagem é primeiro contato da vítima queimada com o setor saúde, sendo o ponto de apoio e de fundamental relevância para o prognóstico terapêutico. Medidas de APH pela enfermagem podem mitigar a evolução do quadro, diminuindo não só a necessidade de atendimentos mais intensivos, assim como reduzindo os custos de saúde ${ }^{7-10}$.

Diante dessa problemática, esse estudo justifica-se pela necessidade de atualização e capacitação da enfermagem no APH às vítimas de queimaduras, pois o prognóstico terapêutico tende a melhorar quanto antes e mais adequada for a assistência prestada. Consequentemente, a população que carece desses cuidados será beneficiada com um atendimento mais acurado.

Ademais, espera-se que esse estudo venha a contribuir com a academia científica, vindo a posteriori servir de base para novas pesquisas, como guia de enfoque para os cuidados pré-hospitalares de enfermagem, enriquecendo assim a literatura disponível. Diante disso, este estudo tem como objetivo identificar as evidências científicas sobre a assistência de enfermagem no atendimento pré-hospitalar a vítima de queimadura.

\section{MÉTODO}

O estudo em questão trata de uma revisão de escopo (scoping review), realizada em setembro de 2020, seguindo o método do Joanna Briggs Institute ${ }^{11}$. Esse delineamento de pesquisa busca reconhecer e mapear as principais evidências acerca de uma determinada área, explorando lacunas investigativas e oferecendo uma visão geral dos principais conceitos sobre o campo de conhecimento ${ }^{11}$. A pesquisa passou pelas etapas de identificação da questão de pesquisa; busca por estudos pertinentes; seleção dos artigos; extração dos dados da pesquisa e agrupamento dos resultados. Esta revisão de escopo foi registrada na plataforma para registro de trabalhos científicos Open Science Framework (https://osf.io/pm7ng) ${ }^{12}$.

Com a finalidade de realizar um rastreamento, bem como a identificação de outras revisões de escopo ou protocolos similares, foi executada uma busca nas plataformas: Open Science Framework (OSF); The Crochrane Library; JBI Clinical Online Network of Evidence for Care and Therapeutics (ConNECT+); Database of Abstracts of Reviews of Effects (DARE); Prospective Register of Systematic Reviews (PROSPERO). Após as buscas, foi possível revelar a inexistência de materiais semelhantes.

A pergunta de pesquisa foi construída utilizando a estratégia PCC, que aponta como mnemônicos fundamentais o: P - População; C - Conceito e C - Contexto. Desse modo, foram estabelecidos os elementos: $\mathrm{P}=$ vítimas de queimaduras; $\mathrm{C}=$ assistência de enfermagem a vítimas de queimadura e $\mathrm{C}=$ atendimento pré-hospitalar. Diante disso, 
foi elaborada a seguinte pergunta de pesquisa: "Como ocorre a assistência de enfermagem no atendimento préhospitalar a vítimas de queimaduras?".

Definida a pergunta de pesquisa, seguiu-se para a busca dos estudos relevantes realizada através do portal de Periódicos da Coordenação de Aperfeiçoamento de Pessoal de Nível Superior (CAPES), no qual foi consultado o acervo das bases de dados da Literatura Latino-Americana e do Caribe em Ciências da Saúde (LILACS), Cochrane Library, Cumulative Index of Nursing and Allied Health (CINAHL), The Scientific Electronic Library Online (SciELO), Web of Science e National Library of Medicine (PubMed). O cruzamento nas bases de dados foi realizado com os descritores "Enfermagem", "Queimaduras" e "Serviços médicos de emergência" indexados no Descritores em Ciência da saúde (DeCS); e "Nursing", "Burns" e "Emergency medical services", em consonância com o Medical Subject Headings (MeSH). Utilizando os operadores booleanos AND e OR.

A fim de ampliar as buscas, para a amostra de resultados, os cruzamentos foram executados com os descritores em inglês, como exposto na figura 1 . Os agrupamentos e seleção de artigos foram realizados nas bases de dados mencionadas anteriormente.

\begin{tabular}{|c|c|}
\hline FONTES DE DADOS & SINTAXE \\
\hline CINAHL & $\begin{array}{l}\text { (burns AND nursing) AND (burns AND emergency medical services) AND (burns AND emergency medical } \\
\text { services AND nursing) AND (emergency medical services AND burns AND Nursing) OR (burns AND nursing) }\end{array}$ \\
\hline COCHRANE & $\begin{array}{l}\text { "burns" in All Text AND "nursing" in All Text "burns" in All Text AND "emergency medical services" in All Text } \\
\text { "burns" in All Text AND "emergency medical services" in All Text AND "nursing" in All Text "burns" in All Text } \\
\text { OR "nursing" in All Text "burns" in All Text OR "emergency medical services" in All Text "burns" in All Text } \\
\text { OR "emergency medical services" in All Text OR "nursing" in All Text }\end{array}$ \\
\hline LILACS & $\begin{array}{l}\text { tw:((tw:("burns")) AND (tw:("nursing"))) tw:((tw:("burns")) AND (tw:("emergency medical services"))) } \\
\text { tw:((tw:("burns")) AND (tw:("emergency medical services")) AND (tw:("nursing"))) tw:((tw:("burns")) OR } \\
\text { (tw:("emergency medical services"))) (tw:("burns")) OR (tw:("emergency medical services")) OR } \\
(\text { tw:("nursing")) }\end{array}$ \\
\hline PUBMED & $\begin{array}{l}\text { ((((Burns AND Emergency medical services AND nursing)) AND (Nursing AND Burns OR Emergency medical } \\
\text { services))) AND ((((((burns AND nursing)) AND (burns AND emergency medical services)) AND (burns AND } \\
\text { emergency medical services AND nursing)) AND (emergency medical services AND burns AND Nursing)) OR } \\
\text { (burns AND nursing)) }\end{array}$ \\
\hline SCIELO & $\begin{array}{l}\text { ("burns") AND ((nursing OR nursing care)) ("burns") AND ((emergency medical services OR emergency)) } \\
\text { ("burns") AND ((emergency medical services OR emergency)) AND ((nursing OR nursing care)) ("burns") OR } \\
\text { ((nursing OR nursing care)) ("burns") OR ((emergency medical services OR emergency)) ("burn") OR } \\
\text { ((emergency medical services OR emergency)) OR ((nursing OR nursing care)) }\end{array}$ \\
\hline Web Of Science & $\begin{array}{l}T S=(\text { Burns) } A N D T S=(\text { Emergency medical services }) \text { OR } T S=(\text { Burns }) \text { AND TS }=(\text { Emergency medical services }) \text { AND } \\
T S=(\text { Nursing) OR TS=(Burns) AND TS=(Emergency medical services) AND TS=(Nursing) }\end{array}$ \\
\hline
\end{tabular}

FIGURA 1: Quadro da sintaxe de pesquisa nas fontes de dados científicas. Natal, RN, Brasil, 2020

Fonte: dados da pesquisa.

Para seleção dos estudos adotou-se como critérios de inclusão artigos disponíveis online gratuitamente na íntegra, publicados nos idiomas português, inglês e espanhol e no recorte temporal de 2016 a 2020. Foram excluídos os trabalhos científicos que fujam do tema proposto pelo estudo e os repetidos mantidos apenas um.

Os artigos foram escolhidos previamente após leitura dos títulos e resumos. A posteriori, foram executadas a leitura e análise textual na íntegra em conformidade com os critérios de inclusão, para compor a amostra final de 12 artigos para extração de informações.

A busca foi executada conjuntamente por dois colaboradores independentes durante o período de coleta usando computadores distintos, a fim de assegurar que nenhum estudo importante seja eliminado. Para evitar incompatibilidades, os estudos foram escolhidos posteriormente a discussão por pares. Depois da seleção, os estudos foram categorizadas em: autores, ano, local de publicação, tipo de estudo, nível de evidência e grau de recomendação em consonância com Oxford Centre for Evidence-based Medicine ${ }^{13}$, população,faixa etária, objetivo e assistência de enfermagem. 


\section{RESULTADOS}

Foram identificados 5225 artigos através dos cruzamentos realizados nas fontes de dados. Após a aplicação dos critérios de inclusão e exclusão, foram descartadas 49 duplicatas e feito a leitura de 417 títulos e resumos, onde foi averiguado que 383 artigos não atendiam aos critérios estabelecidos. Após esse processo, evidenciado na figura 2, um total de 12 artigos foram incluídos para a elaboração dos resultados.

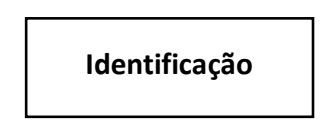

Triagem

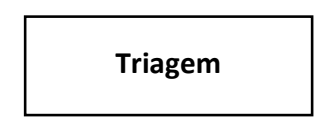

Elegibilidade

Inclusão

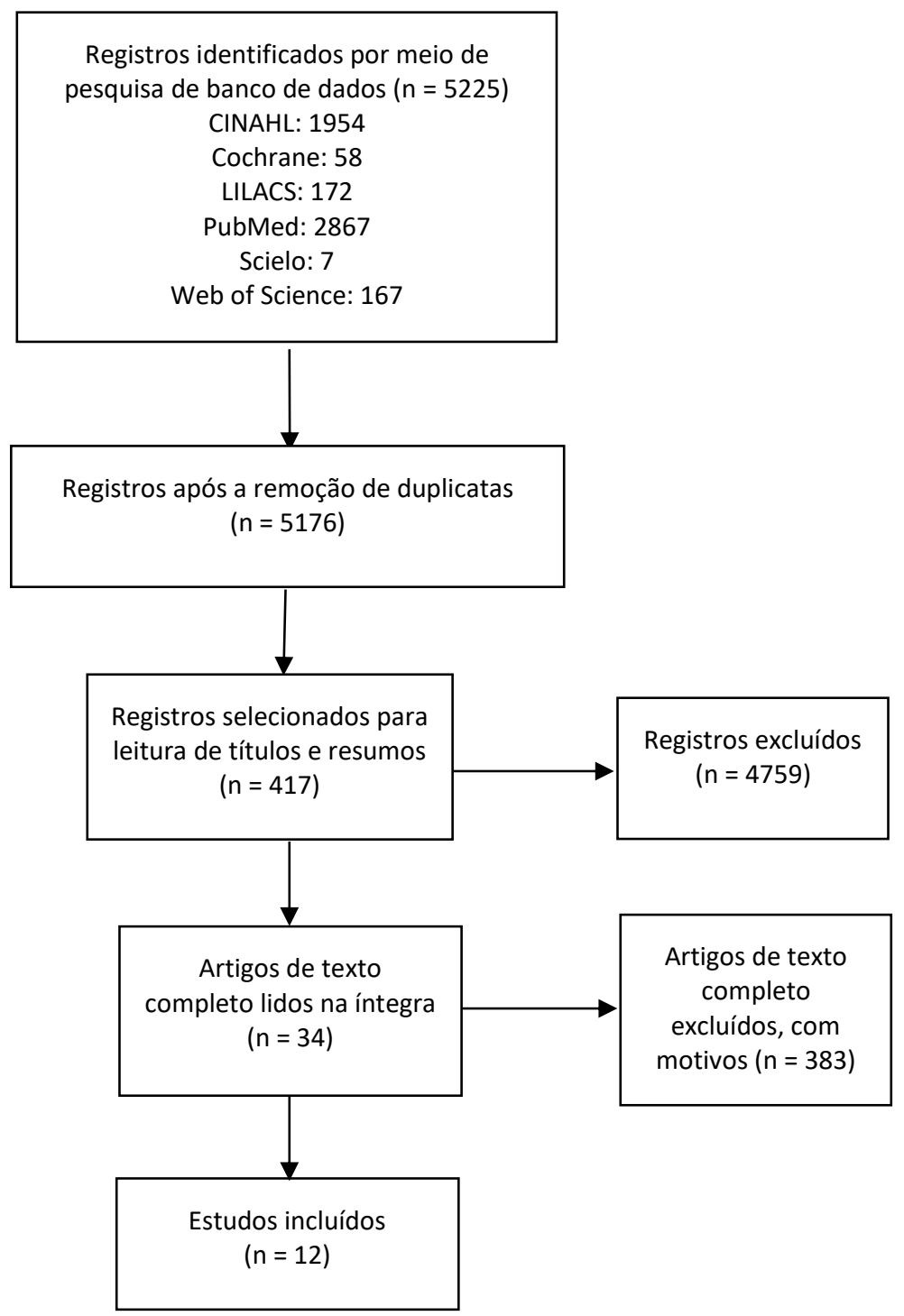

FIGURA 2: Diagrama de fluxo PRISMA-ScR (adaptado). Natal, RN, Brasil, 2020 Fonte: dados da pesquisa.

Os 12 estudos incluídos, sintetizados na figura 3, foram publicados entre os anos de 2016 a 2019. Dentre eles, os países com maior número de publicações foram: o Brasil com seis estudos $(50,0 \%)^{14,18,20-21,23-24}$, Estados Unidos da América com 3 publicações $(25,0 \%)^{15,19,25}$, Argentina, Equador e Tanzânia com uma publicação cada $(25 \%)^{16-17,22}$. Sendo eles: um guia de prática clínica ${ }^{22}$, três revisões integrativas ${ }^{20-21,24}$, uma revisão de literatura ${ }^{23}$, um estudo de caso ${ }^{18}$, três estudos descritivos ${ }^{14,16,19}$, uma revisão retrospectiva ${ }^{15}$ e um estudo qualitativo descritivo ${ }^{17}$.

Acerca do nível de evidência e grau de recomendação, foi utilizada as orientações estabelecidas pela Oxford Centre for Evidence-based Medicine. Destaca-se que o maior nível de evidência corresponde ao menor número indicado, e quanto ao grau de recomendação o " $A$ " é apontado como mais recomendado e o " $D$ " como menos recomendado ${ }^{13}$. 
O local de publicação dos estudos inclui países de vários continentes. A população envolve crianças, jovens, adultos e principalmente enfermeiros, contudo, dois artigos não especificaram sua população-alvo. O nível de evidência variou entre 2B e 4, já o grau de recomendação ficou entre B e C. Quanto aos objetivos encontrados na amostra, avaliam predominantemente o conhecimento dos enfermeiros sobre o atendimento, descrição e implementação da assistência de enfermagem às vítimas com queimaduras. Dentre as assistências de enfermagem mais encontrados destaca-se a reposição volêmica com infusão de fluidos, resfriamento local com água limpa, corrente e em temperatura ambiente, monitoramento e controle dos Sinais Vitais (SSVV), principalmente controle da respiração com uso de oxigenoterapia, analgesia e cuidados com a ferida, como o uso de coberturas oclusivas, identificação do agente causador, avaliação da Superfície Corporal Queimada (SCQ), extensão e profundidade da lesão.

\begin{tabular}{|c|c|c|}
\hline $\begin{array}{l}\text { *ID/ Ano/Local de } \\
\text { publicação }\end{array}$ & $\begin{array}{l}\text { Tipo de estudo/ Nível de } \\
\text { evidência/ Grau de } \\
\text { recomendação/População }\end{array}$ & Assistência de enfermagem \\
\hline E1 $14 / 2016 /$ Brasil & $\begin{array}{l}\text { Estudo descritivo/ } \\
\text { 2B/B/Infanto-juvenil }\end{array}$ & $\begin{array}{l}\text { Afastar a vítima do agente causador, resfriar com água limpa e corrente } \\
\text { por 10-20 minutos, aquecer a vítima. }\end{array}$ \\
\hline E2 $15 / 2017 / E U A$ & $\begin{array}{l}\text { Revisão retrospectiva/ } \\
2 \mathrm{~B} / \mathrm{B} / \mathrm{Ne}^{*}\end{array}$ & $\begin{array}{l}\text { Reposição volêmica, monitorar sinais vitais (SSVV), avaliar sinais de lesão } \\
\text { por inalação. }\end{array}$ \\
\hline E3 $^{16} / 2019 /$ Equador & $\begin{array}{c}\text { Estudo } \\
\text { descritivo/2B/B/Enfermeiros }\end{array}$ & Manter a pele afetada úmida, reposição volêmica. \\
\hline E4 ${ }^{17} / 2019 /$ Tanzânia & $\begin{array}{c}\text { Estudo qualitativo } \\
\text { descritivo/2B/B/Enfermeiros }\end{array}$ & Aplicação de água fria, fazer curativo oclusivo. \\
\hline E5 $18 / 2018 /$ Brasil & Estudo de caso/4/C/Adultos & $\begin{array}{l}\text { Avaliar extensão da lesão. Monitorar SSVV, saturação de O2, risco de } \\
\text { infecção e viabilizar Acesso Venoso Periférico (AVP). }\end{array}$ \\
\hline E6 $19 / 2016 / E U A$ & $\begin{array}{c}\text { Estudo } \\
\text { descritivo/2B/B/Enfermeiros }\end{array}$ & $\begin{array}{l}\text { Fazer reposição volêmica e analgesia. Observar sinais de infecção e má } \\
\text { perfusão arterial periférica. }\end{array}$ \\
\hline E720/2017/Brasil & $\begin{array}{c}\text { Revisão } \\
\text { integrativa/4/C/Adultos }\end{array}$ & $\begin{array}{l}\text { Lavar região afetada, analgesia com medidas farmacológicas e não } \\
\text { farmacológicas, avaliar casos de queimaduras por inalação. }\end{array}$ \\
\hline E8 $21 / 2016 /$ Brasil & $\begin{array}{c}\text { Revisão } \\
\text { integrativa/4/C/Enfermeiros }\end{array}$ & $\begin{array}{l}\text { Monitorar a saturação de } 02 \text { e SSVV. Manter vias aéreas pérvias, usar } \\
\text { clorexidina para limpeza, realizar curativo, identificar edema, aplicar } \\
\text { compressas e fazer analgesia e AVP. }\end{array}$ \\
\hline E922/2016/Argentina & $\begin{array}{c}\text { Guia de prática } \\
\text { clínica/4/C/Crianças }\end{array}$ & $\begin{array}{l}\text { Lavar a região afetada, verificar SSVV, administrar O2 e analgésicos. } \\
\text { Repor volemia, limpar lesão com clorexidina } 4 \% \text {, usar nitrofurazona } \\
0,22 \% \text { ou sulfadiazina de prata. }\end{array}$ \\
\hline E1023/2017/Brasil & $\begin{array}{c}\text { Revisão de } \\
\text { literatura/2C/B/Ne* }\end{array}$ & $\begin{array}{l}\text { Repor fluidos, viabilizar AVP, limpar lesão com SF } 0,9 \% \text { ou água. Aplicar } \\
\text { curativos estéreis, observar SSVV e sinais de hipoxemia, hipotermia, } \\
\text { sudorese, cianose e hipovolemia. }\end{array}$ \\
\hline E1124/2018/Brasil & $\begin{array}{c}\text { Revisão } \\
\text { integrativa/4/C/Adultos }\end{array}$ & $\begin{array}{l}\text { Remover agente químico em pó com escovas/panos, resfriar com água } \\
\text { corrente, retirar adornos/roupas, repor volemia. }\end{array}$ \\
\hline E1225/2018/EUA & $\begin{array}{c}\text { Estudo } \\
\text { descritivo/2B/B/Enfermeiros }\end{array}$ & $\begin{array}{l}\text { Cuidados com a ferida, controle de infecção, preservar segurança do } \\
\text { paciente. }\end{array}$ \\
\hline
\end{tabular}

FIGURA 3: Quadro de síntese dos resultados encontrados na amostra final. Natal, RN, Brasil, 2020.

Ne*: Não especificado. EUA: Estados Unidos da América. *ID: identificação dos estudos.

\section{DISCUSSÃO}

Queimaduras são traumas de custos elevados com tratamento e recuperação, além de serem muitas vezes debilitantes e incapacitantes, dificultando a aceitação e prognóstico pela vítima. Profissionais que atuam nessa área precisam estar capacitados o suficiente para atuarem nas especificidades de cada lesão. Esse preparo aliado a agilidade é de fundamental no atendimento precoce do público-alvo ${ }^{26}$.

Um método simples, de baixo custo e amplamente utilizado pela enfermagem no APH em queimaduras é o resfriamento no local da lesão. Nesta revisão foram destacados diretamente em $58,3 \%$ dos estudos a importância do uso de água corrente, limpa, a temperatura ambiente e por tempo pré-estabelecido (não inferior a 10 minutos), como uma das primeiras condutas do APH em queimados. A demora no resfriamento local pode acarretar agravamento no 
quadro da queimadura e necessitar de períodos maiores de atendimento hospitalar, além de promover a intuitiva redução na temperatura, reduz as chances de infecção local, promove analgesia e cicatrização mais rápida em queimaduras profundas ${ }^{7}$.

Outra assistência é a reposição volêmica, sendo uma das bases do APH em queimaduras que repercute positivamente quando executada adequada e o mais precocemente possível. A fórmula de Parkland, frequentemente usada para calcular a reposição volêmica em pacientes queimados traz que, em adultos deve-se considerar a reposição de $(4 \mathrm{ml}$ x peso corporal em $\mathrm{kg} \times$ porcentagem de SCQ), sendo que $50 \%$ do resultado deverá ser administrado nas primeiras 8 horas após a lesão e a outra metade será administrada nas outras 16 horas restantes. Ou seja, esse cálculo é feito tomando-se por noção os líquidos que por ocasião do trauma a vítima perdeu e ainda considerando o que a mesma venha a perder total nas primeiras 24 horas pós-queimadura. Cerca de $66,6 \%$ dos artigos apontam a reposição volêmica como assistência de enfermagem primordial, sendo realizada por meio de punção de AVP ou AVC e administração de fluidos segundo prescrição médica ${ }^{2,26}$.

Por diversos benefícios, o curativo oclusivo é considerado uma medida amplamente utilizada em queimaduras. Deve conter cobertura específica conforme o aspecto da lesão e objetivo a ser alcançado, além de possuir espessura suficiente para absorver os fluidos liberados pela ferida, proteção contra infecções externas e garantir controle da temperatura local. Corrobora com tais dados 33,3\% da amostra, que trazem em seus resultados o uso do curativo oclusivo como assistência de enfermagem utilizada em queimaduras ${ }^{27}$.

Curativos a base de prata, principalmente a sulfadiazina de prata $1 \%$ são métodos de primeira escolha em curativos para queimaduras. Suas vantagens são o auxílio na reepitelização, controle antimicrobiano e analgesia. Além disso, se encontram disponíveis no mercado, os curativos hidrocolóides, hidrogéis, Ácidos Graxos Essenciais (AGE), gazes não aderentes, membranas sintéticas e biológicas, matriz de regeneração dérmica, malha de algodão parafinado, dentre outros. Os curativos tem potencial para transformar uma lesão aberta e parcialmente contaminada numa lesão limpa. Lembrando que a oclusão deve ser evitada apenas em queimaduras em regiões genitais e faciais ${ }^{28}$.

Logo, no APH em queimaduras é fundamental realizar a avaliação da extensão, agente causador e profundidade da lesão. Aproximando-se de uma vítima queimada é necessário investigar qual a fonte causadora da lesão e seu tempo de exposição. Depois, afasta-se a vítima do fator agressor e retirar-lhe as roupas e adornos, atentando para as particularidades de cada agente causador. Apenas 33,3\% da amostra se deteve a explicitar essa assistência de enfermagem em seus resultados ${ }^{29}$. Vale ressaltar, que as intervenções realizadas podem variar de acordo com o tipo de agente térmico envolvido na lesão, influenciando assim na assistência prestada.

Vítimas fatais de incêndios geralmente têm como causa mortis a inalação de fumaça quente e altamente tóxica. Essa fumaça acaba provocando queimaduras internas, principalmente no trato respiratório. Isso pode rebaixar rapidamente o nível de consciência por hipóxia e óbito por asfixia. Nota-se em $58,3 \%$ dos resultados a necessidade de monitoramento dos SSVV com foco na respiração ${ }^{29}$.

A equipe de enfermagem deve sempre que possível, fazer a avaliação primária imediata, que não deve durar mais que 5 min e avaliação secundária, centrando-se na manutenção da permeabilidade das vias aéreas e controle dos SSVV. O oxigênio deverá ser fornecido a $100 \%$ via máscara umidificada e na hipótese de intoxicação por monóxido de carbono (CO), a terapia segue durante $3 \mathrm{~h}$ com a mesma dosagem. Vias aéreas devem ser aspiradas e investigadas quanto a presença de corpos estranhos. A cabeceira será elevada a 30ㅇ e a região cervical mantida em hiperextensão. Avaliar e comunicar presença de dispneia, cianose e tosse ${ }^{30}$.

Quanto mais grave a queimadura, maiores são as chances de instabilidade nos parâmetros vitais. Daí a importância do monitoramento constante dos SSVV. A pele lesada, sem a epiderme, tende a não controlar a retenção de calor corporal. Deve-se manter o paciente aquecido com mantas térmicas ou cobertores. $\mathrm{O}$ uso do oxímetro de pulso não é recomendado, visto que o mesmo pode mascarar a intoxicação por CO. Alterações na respiração como ruídos, dispneia, incursões respiratórias ineficazes observadas pela não expansão total do tórax, refletindo em aumento da frequência respiratória e cardíaca são também indícios de queimaduras por inalação. Em queimaduras de extremidades há a formação de edema por extravasamento local de líquidos, o que dificulta a aferição precisa da pressão arterial periférica durante o APH. A dor deverá ser analisada com rigor, assim como os outros SSVV, pois está intimamente ligada com a estabilidade dos outros parâmetros ${ }^{2,31}$.

Por possuir muitas terminações nervosas, a pele quando queimada acaba lesionando essas terminações, o que gera muita dor. Cada indivíduo possui um limiar de dor e isto reflete na assistência de enfermagem por motivos da dor repercutir nos aspectos físicos, biológicos e emocionais. Dos estudos revisados, $41,6 \%$ detiveram-se a importância do controle analgésico no APH pelo enfermeiro em atendimento a vítimas queimadas. Destarte, mostra-se a carência para mais estudos que objetivem assistir não só a ferida, mas a dor que ela carrega ${ }^{32}$. 
Analisando-se o nível de evidência e o grau de recomendação dos estudos selecionados, pode-se concluir uma maior frequência de nível de evidência valor 2B, com um total de seis estudos (50\%) da amostra. Um estudo apresenta nível 2C (8,3\%) e cinco são de nível 4 (41,6\%). Relacionado ao grau de recomendação, sete estudos são de grau B (58,3\%) e cinco se classificam em grau C (41,6\%). Segundo a Oxford Centre for Evidence-based Medicine ${ }^{13}$ tais resultados revelam uma baixa força no nível de evidência e grau de recomendação da amostra, extraindo dessa informação a necessidade no desenvolvimento de pesquisas mais expressivas.

Diante do exposto nos resultados e discussão, considerando a relevância da questão e o número elevado de traumas por queimaduras mundialmente, essa revisão de escopo fornece subsídios para enriquecimento da literatura científica disponível, descrevendo como está sendo desenvolvida a assistência de enfermagem no APH as vítimas de queimaduras. Evidenciou-se nos achados um maior destaque, em porcentagem, para a reposição volêmica (66,6\%), resfriamento local (58,3\%), controle dos SSVV, principalmente o controle respiratório (58,3\%), analgesia (41,6\%) e cuidados com a ferida (33,3\%).

Contudo, essa revisão limita-se a estudos publicados recentemente, no período de 2016 a 2020. Esse limite temporal pode ter restringido alguns artigos de maior relevância, porém, tentou-se ao máximo buscar por obras que atendessem aos critérios de melhor seleção e nas bases de dados mais acessíveis. Resultado disso é que a amostra comporta estudos de vários países e com populações diversas.

\section{CONCLUSÃO}

A população envolvida foram principalmente enfermeiros que atuam no setor de atendimento de queimados. Os objetivos das publicações em análise elencaram e descreveram a assistência efetivada pelos enfermeiros em atuação. Por apresentarem nível de evidência de $2 \mathrm{~B}$ a 4 e um grau de recomendação entre $\mathrm{B}$ e $\mathrm{C}$, a amostra expôs uma rasa expressividade acadêmica. Em remate, os resultados puderam evidenciar os cuidados principalmente com base no resfriamento local da lesão, reposição volêmica, uso de curativos oclusivos, avaliação das características da ferida, monitoramento dos SSVV com observância maior na avaliação respiratória e controle da dor.

Com isso, a partir da análise dos estudos, foi possível observar que a assistência do enfermeiro frente ao atendimento pré-hospitalar às vítimas de queimaduras, de primeiro a quarto grau, se dá principalmente pelo o controle da evolução dos danos causados pelo incidente até o momento da chegada ao atendimento terciário.

Portanto, espera-se que o presente estudo sirva para elucidar o conhecimento científico acerca das principais assistências de enfermagem prestadas a pacientes vítimas de queimaduras atendidas pelos serviços de emergência, a fim de contribuir para a qualidade dos cuidados prestados, e que venha a despertar o interesse cada vez maior para a produção de novas pesquisas científicas na área e com maior grau de expressividade acadêmica.

\section{REFERÊNCIAS}

1. Moreto RW. Cuidados de enfermagem no atendimento pré-hospitalar à vítima de queimaduras. In: Anais do Congresso Nacional de Iniciação Científica CONIC- SEMESP; 2017, São Paulo, Brasil. São Paulo (SP); 2017 [cited 2020 Apr 08]. Available from: http://conic-semesp.org.br/anais/files/2017/trabalho-1000024370.pdf.

2. National Association of Emergency Medical Technicians-NAEMT. Lesões por queimadura. In. Atendimento pré-hospitalar traumatizado -PHTLS. São Paulo: Edições 8/Elsevier; 2017. p.406-28.

3. Organização Mundial da Saúde. Violence and Injury Prevention: Burns. OMS; 2015 [cited 2020 Mar 11]. Available from: https://www.who.int/violence_injury_prevention/other_injury/burns/en/.

4. Ministério da Saúde (Br). Queimados [Internet]. Brasília (DF): Ministério da Saúde; 2017 [cited 2020 Mar 11]. Available from: https://www.saude.gov.br/component/content/article/842-queimados/40990-queimados.

5. Organização Mundial da Saúde. Burns. OMS; 2018 [cited 2020 Mar 11]. Available from: https://www.who.int/en/newsroom/fact-sheets/detail/burns.

6. Dias LPR, Mendes RS, Trigueiro GP, Assis EV, Feitosa ANA, Sousa MNA. Nursing in pre-hospital care: paper, occupational hazards and consequences. Rev. Interdisciplinar em Saúde. 2016 [cited 2020 Apr 08]; 3(1):223-36. Available from: http://interdisciplinaremsaude.com.br/Volume_9/Trabalho_13.pdf.

7. Bourke $P$, Bison AVF. The importance of cooling a burn injury in the pre hospital setting. Rev Brasileira de Queimaduras. 2015 [cited 2020 Apr 25]; 14(1):31-34. Available from: http://rbqueimaduras.org.br/details/240/pt-BR/a-importancia-doresfriamento-da-queimadura-no-atendimento-pre-hospitalar.

8. Ribeiro AC, Silva YB. Pre-hospital nursing in basic life support: ethical and legal postulates of the profession. Cogitare Enfermagem. 2016 [cited 2020 Apr 09]; 21(1):01-08. DOI: http://dx.doi.org/10.5380/ce.v21i1.42118.

9. Rosa PH, Vieira TG, Ilha S, Antunes BS. The treatment of burns in the emergency service: the nurse in this context. Rev. eletrônica Disciplinarum Scientia. 2018 [cited 2020 Apr 26]; 19(3): 525-36. Available from: https://periodicos.ufn.edu.br/index.php/disciplinarumS/article/view/2709/2263.

10. Ismael ICG. The importance of the role of nursing in the assistance process in patients with burns. Rev. Eletrônica Acervo Saúde. 2019 [cited 2020 Apr 07]; (23):e209. DOI: https://doi.org/10.25248/reas.e209.2019. 
11. Peters MDJ, Godfrey C, McInerney P, Munn Z, Tricco AC, Khalil, H. Chapter 11: Scoping Reviews (2020 version). In: Aromataris E, Munn Z (Editors). Joanna Briggs Institute Reviewer's Manual, JBI [Internet]. 2020 [cited 2020 Sep 04]. Available from: https://reviewersmanual.joannabriggs.org/.

12. Silva RIM, Oliveira ES, Rocha RRA, Costa TMS, Dantas DV. Assistência de enfermagem no atendimento pré-hospitalar a vítimas de queimaduras: scoping review. 2020 [cited 2020 May 24]. Available from: https://osf.io/pm7ng.

13. Centre For Evidence- Based Medicine. Levels of Evidence (March 2009). 2009 [cited 2020 Abr 09]. Available from: https://www.cebm.net/2009/06/oxford-centre-evidence-based-medicine-levels-evidence-march-2009/.

14. Brito JG, Martins CBG. Domestic burns among children, adolescents and young adults: urgency and emergency cases. Rev. Eletrônica de Enfermagem. 2016 [cited 2020 Apr 27]; 18:e1139. DOI: https://doi.org/10.5216/ree.v18.32141.

15. Caldwell NW, Melvin MLS, Chung KK, Salinas J, Shiels ME, Cancio LC, et al. Follow-Up Evaluation of the U.S. Army Institute of Surgical Research Burn Flow Sheet for En Route Care Documentation of Burned Combat Casualties. Military Medicine. 2017 [cited 2020 Apr 27]; 182(11-12):e2021-e2026. DOI: https://doi.org/10.7205/MILMED-D-17-00121.

16. Fernández LP, González ARA, Cedeño MMC. Nursing care in pediatric patients with burns and effects due to separation of the family bond. Rev. de Enfermería y Humanidades- Cultura de los Cuidados. 2019 [cited 2020 Apr 27]; (54):374-83. DOI: https://doi.org/10.14198/cuid.2019.54.32.

17. Marwa NP, Tarimo EAM. Provision of care to hospitalized pediatric burn patients: a qualitative study among nurses at Muhimbili National Hospital, Dar es Salaam, Tanzania. BMC Nursing. 2019 [cited 2020 Apr 27]; 18(8). DOI: https://doi.org/10.1186/s12912-019-0335-1.

18. Neto VLS, Costa RTS, Lucena EA, Silva SC, Pereira VM, Silva RAR. Implementing the nursing process for a burn patient: a case study. Rev. enferm. UERJ. 2018 [cited 2020 Apr 27]; 26: e30962. DOI: https://doi.org/10.12957/reuerj.2018.30962.

19. Olszewski A, Yanes A, Stafford J, Greenhalgh DG, Palmieri TL, Sen S, et al. Development and implementation of an innovative burn nursing handbook for quality improvement. Journal of Burn Care Research. 2016 [cited 2020 Apr 27]; 37(1):20-4. DOI: https://doi.org/10.1097/BCR.0000000000000299.

20. Pinho FM, Sell BT, Sell CT, Senna CVA, Martins T, Fonseca ES, et al. Nursing care of adult burn patient: an integrative review. Rev. Brasileira de Queimaduras. 2017 [cited 2020 Apr 27]; 16(3):181-7. Available from: http://www.rbqueimaduras.com.br/details/391/pt-BR.

21. Pinho FM, Amante LN, Salum NC, Silva R, Martins T. Nursing care guideline actions in burnt adult patients. Rev. Brasileira de Queimaduras. 2016 [cited 2020 Apr 26]; 15(1):13-23. Available from: http://rbqueimaduras.org.br/details/288/pt-BR/guidelinedas-acoes-no-cuidado-de-enfermagem-ao-paciente-adulto-queimado.

22. Salvatierra VD. Nursing interventions in the initial care of the burn pediatric patient. Visión enfermería actualizada. 2016 [cited 2020 Apr 27]; 13(47):29-37. Available from: http://docs.bvsalud.org/biblioref/2019/08/1008585/vea_47-29-37.pdf.

23. Santos CA, Santos AA. Nursing care in prehospital care for the burned patient: a review of the literature. Rev. Brasileira de Queimaduras. 2017 [cited 2020 Apr 27]; 16(1):28-33. Available from: http://rbqueimaduras.org.br/details/344/ptBR/assistencia-de-enfermagem-no-atendimento-pre-hospitalar-ao-paciente-queimado--uma-revisao-da-literatura.

24. Valente TM, Nascimento MFA, Júnior FRS, Souza JPF, Martins CB, Valente TM, et al. Importance of an effective pre-hospital care for victims of burns: an integrating review. Rev. Brasileira de Queimaduras. 2018 [cited 2020 Apr 27]; 17(1):50-55. Available from: http://www.rbqueimaduras.com.br/details/417/pt-BR/importancia-de-um-atendimento-pre-hospitalar-efetivo-aadultos-vitimas-de-queimaduras--uma-revisao-integrativa.

25. Vanfosson CA, Jones TL, Yoder LH. Monthly variation of unfinished nursing care at the US Army Burn Center. Burns. 2018 [cited 2020 Apr 27]; 44(8):1910-9. DOI: https://doi.org/10.1016/j.burns.2018.03.008.

26. Cunha LVT, Júnior FJAC, Santiago DO. Primary care of burned patient: an assessment of knowledge of medical internship students. Rev. Brasileira de Queimaduras. 2016 [cited 2020 May 06]; 15(2):80-6. Available from: http://www.rbqueimaduras.com.br/details/298/pt-BR.

27. Tavares WS, Silva RS. Dressing used in the treatment of burns: an integrative review. Rev. Brasileira de Queimaduras. 2015 [cited 2020 May 06]; 14(4):300-6. Available from: http://rbqueimaduras.org.br/details/282/pt-BR.

28. Oliveira APBS, Peripato LA. The ideal coverage for treatment in a burning patient: an integrating literature review. Rev. Brasileira de Queimaduras. 2017 [cited 2020 May 19]; 16(3):188-93. Available from: http://www.rbqueimaduras.com.br/details/392/pt-BR.

29. Corpo de Bombeiros Militar do Estado de Goiás. Emergências Traumáticas. In: Manual Operacional de Bombeiros- Resgate PréHospitalar. Goiânia (GO): CBMGO; 2016 [cited 2020 May 07]; seção 21. Available from: https://www.bombeiros.go.gov.br/wpcontent/uploads/2015/12/MANUAL-DE-RESGATE-PR\%C3\%89-HOSPITALAR.pdf.

30. Pinho FM, Klein TCR. Elaboração de Procedimento Operacional Padrão (POP) sobre avaliação de pacientes com feridas agudas não cirúrgicas: QUEIMADURAS para o Hospital Universitário professor Polydoro Ernani de São Thiago. 2017 [cited 2020 May 19]; versão 1: 4-5. Available from: http://www.hu.ufsc.br/pops/pop-externo/download?id=208.

31. Coelho EFP, Pereira MGN. The nurse's action in the management of pain in the emergency and emergency room. Rev Internacional de Apoyo a la Inclusión, Logopedia, Sociedad y Multiculturalidad. 2017 [cited 2020 May 19]; 3(4):73-86. Available from: https://revistaselectronicas.ujaen.es/index.php/riai/article/view/4291.

32. Silva LD, Henrique DM, Maia PG, Almeida ACL, Nascimento NM, Gomes PP, et al. Patient nursing care large burned subject to sedation and analgesia: a literature review. Revista Nursing. 2018 [cited 2020 May 08]; 21(236):2021-6. Available from: https://pesquisa.bvsalud.org/portal/resource/pt/biblio-907861. 